\title{
Evaluation of the Use of Titanium and Silicon Nanoparticles for the Improvement the Thermal Conditioning System in Vehicles
}

\author{
Jaime Cueva ${ }^{1}$, Cristian Yalama ${ }^{2}$, Fredy Llulluna ${ }^{3}$, Myrian Larco ${ }^{4}$, Claudio Cordova \\ ${ }^{1,2}$ Department of Mechanical Engineering / Universidad Técnica de Ambato \\ Av. Los Chásquis 180207, Ambato, Ecuador \\ jcueva4473@uta.edu.ec; cyalama4589@uta.edu.ec \\ ${ }^{3,4}$ Department Sustainable Architecture / Universidad Regional Amazónica Ikiam \\ Parroquia Muyuna, kilómetro 7 vía a Alto Tena. Napo, Ecuador \\ fredy.llulluna@ikiam.edu.ec; myrian.larco@ikiam.edu.ec \\ ${ }^{5}$ Department of Basic Sciences /Escuela Politécnica Nacional \\ Ladrón de Guevara E11-253, Quito, Ecuador \\ claudio.cordova@epn.edu.ec
}

\begin{abstract}
This resecahc present, the improved cooling system of a vehicle has been developing research of new materials that help dissipate heat inside the engine more efficiently, it is for this reason that in this research project coolants were developed under the ASTM D- 3306 standard using nanofluids with nanoparticles of titanium dioxide ( $93.9 \mathrm{~nm}$ ) and silicon ( $96.3 \mathrm{~nm}$ ) produced by attrition milling, centrifuging and filtering dioxide, which were used in a vehicle gasoline of 1600 cc engine and fuel injection, in order to analyze the thermal and mechanical behavior of the vehicle, compared to commercial coolants on the market, for it touch and power tests, dynamic emissions and fuel consumption ASM were performed. Better engine performance was obtained when using coolants with titanium dioxide and silicon dioxide nanoparticles, the greatest increase was the titanium.
\end{abstract}

Keywords: coolant, nanofluid, nanoparticle, silice, titanium.

\section{Introduction}

Continuous technological development in the automotive industries has increased the demand for high-efficiency engines. A high-efficiency engine relies not only on performance, but also on better fuel economy and lower emissions. There are many systems that influence engine performance such as ignition, fuel, cooling systems, etc.

One of the parameters that affects the performance of the motor is its cooling speed, the addition of fins is one of the solutions that can be implemented to increase the heat transfer since a greater heat transfer area is provided. its coefficient of heat by convection of air, however, the traditional approach of increasing the cooling rate using this method has already reached its limit. [10]

As a result, there is a need to implement new and innovative refrigerant fluids that possess higher thermal conductivity compared to conventional refrigerants such as water, ethylene glycol, and commercial coolants that exhibit very low thermal conductivity. With the advancement of nanotechnology, the new generation of coolants known as "nanofluids" have been developed, finding that these fluids offer higher thermal conductivity compared to conventional coolants. Nanofluids are liquids (water, ethylene glycol, etc.) where nano-sized particles are dispersed, these particles are known as nanoparticles $(<100 \mathrm{~nm})$, which can be metallic or non-metallic. [3] [5]

Nanofluids are a potential replacement for conventional coolants in the engine cooling system. Research has shown an interesting behavior during experiments, which have shown that an increase in thermal conductivity and a higher heat transfer coefficient are obtained. By obtaining an increase in heat transfer using nanofluids as coolants in the engines, it would allow to reduce the size of the engine components such as the radiator, which would allow to obtain a better positioning of the engine, a reduction in costs and more aerodynamic designs, thus achieving improvement engine performance.

When using refrigerants with nanoparticles of alumina, titanium dioxide or silicon dioxide, multi-walled carbon nanoparticles (MWCNT nanotubes), among others, an increase in heat transfer of around 15-40\% is obtained, which translates into a Better aerodynamic function for the design of the front area of the vehicle by reducing the size of the engine by $20 \%$, the friction coefficient could be reduced, and the fuel efficiency could be improved. [1] 


\section{Nanofluids}

Nanofluid is known as the dispersion of nano-sized materials, less than $100 \mathrm{~nm}$ (nanoparticles), in a base fluid (water, ethylene glycol, oils, etc.), forming nanoscale colloidal suspensions. Nanofluids have improved thermophysical properties such as: viscosity, thermal conductivity, thermal diffusibility, and heat transfer coefficients (conductive, convective) compared to the base fluid. They are used in different applications such as: heat transfer, transport, electronics, medicine, among others. Materials such as: [5]

- Oxides $\left(\mathrm{SiO}_{2}, \mathrm{TiO}_{2}, \mathrm{CuO}\right)$.

- $\quad$ Nitrides (AlN, SiN).

- $\quad$ Carbides ( $\mathrm{SiC}, \mathrm{TiC})$.

- $\operatorname{Metals}(\mathrm{Cu}, \mathrm{Ag}, \mathrm{Au})$.

- Semiconductors $(\mathrm{SiC})$.

- Carbon nanotubes.

Nanofluids have a wide variety of applications, they are used in heat transfer, automotive, electronics and biomedical applications. In the automotive industry, greater energy efficiency is required, for which purpose not only higher performance engines are sought, but also lower fuel consumption. Therefore, using nanofluids as coolants, brake fluid and in fuel can reduce the size and weight of vehicle components (radiator and engine) without affecting performance. [6]

The use of nanofluids as coolants would allow a smaller size and better positioning of the radiator in an engine. By having greater efficiency, the coolant pumps and truck engines could be operated at higher temperatures that allow greater power to be obtained while still meeting emission standards (percentages of polluting emissions). The use of nanofluids with high thermal conductivities in radiators can allow the reduction in the frontal area of the radiator by up to $10 \%$, which can lead to fuel savings of up to 5\%, friction and wear can be reduced, reducing parasitic losses in the operation of components such as pumps and compressors. [1] [9]

\section{Properties of nanofluids}

The thermophysical properties of nanofluids determine the thermal and physical behaviour in the presence of external factors (temperature, pressure, etc.). There is no theoretical formula currently available to determine most of the properties of nanofluid. However, Hussein determined the following relationships and results. [3]

\subsection{Concentration by weight or volume}

The concentration by weight $(\Phi)$ is given by:

$$
\Phi=\left(\frac{m_{p}}{m_{p}+m_{f}}\right) * 100
$$

Where:

$m_{p}$ : Mass of nanoparticles [gr]

$m_{f}$ : Mass of the fluid (water) [gr]

The volume concentration $(\Phi)$ is given by

$$
\Phi=\left(\frac{V_{p}}{V_{p}+V_{f}}\right) * 100
$$

Where: 
$V_{p}$ : Volume of nanoparticles [ml]

$V_{f}$ : Volume of the fluid (water) [ml]

\subsection{Density}

The density of the nanofluid $\left(\rho_{n f}\right)$ is expressed as a function of the concentration by weight of the particles and is given by:

$$
\rho_{n f}=\left(\frac{\Phi}{100}\right) \rho_{p}+\left(1-\frac{\Phi}{100}\right) \rho_{f}
$$

Where:

$\rho_{p}$ : Density of the nanoparticles $\left[\mathrm{Kg} / \mathrm{m}^{3}\right]$

$\rho_{f}$ : Density of the fluid (water) $\left[\mathrm{Kg} / \mathrm{m}^{3}\right]$

\subsection{Specific heat}

The specific heat of the nanofluid $\left(C_{n f}\right)$ is expressed as a function of the concentration by weight of the particles and is:

$$
C_{n f}=\frac{\left(\frac{\Phi}{100}\right) \rho_{p} C_{p}+\left(\left(1-\left(\frac{\Phi}{100}\right)\right) \rho_{f} C_{f}\right.}{\rho_{n f}}
$$

Where:

$C_{p}$ : Specific heat of the nanoparticles

$C_{f}$ : Specific heat of the fluid (water)

\subsection{Thermal conductivity}

The thermal conductivity of the nanofluid $\left(k_{n f}\right)$ is:

$$
\frac{k_{n f}}{k_{f}}=\frac{k_{p}+(n-1) k_{f}-(n-1) \Phi\left(k_{f}-k_{p}\right)}{k_{p}+(n-1) k_{f}+\Phi\left(k_{f}-k_{p}\right)}
$$

Where:

$k_{f}$ : Thermal conductivity of the fluid (water) $[\mathrm{w} / \mathrm{mk}]$

$k_{p}$ : Thermal conductivity of the nanoparticle $[\mathrm{w} / \mathrm{m} k]$

$n$ : Factor in form

\section{Materials and methods}

To obtain nanoparticles of titanium dioxide (anatase), $100 \mathrm{~g}$ of the material, $200 \mathrm{~g}$ of $1 \mathrm{~mm}$ zirconium balls and $100 \mathrm{~g}$ of $2.5 \mathrm{~mm}$ diameter zirconium balls were weighed, using the dry milling method. by wear for 20 intervals of 20 minutes, this method was used to obtain a greater quantity of nanoparticles with a shorter process time. The ground material obtained and the balls were separated using the ASTM E-11 N60, N120, N270 sieves. [3] [6] [9]

The characterization of the size and agglomeration of the DLS and AFM particles from the soil was carried out, since the nanometric size was not obtained, a 10\% solids solution was prepared which was centrifuged for 10 minutes at a speed 
of $1840 \mathrm{rpm}$, then fluidized using a $0.1 \mu \mathrm{m}$ Millex filter, the filtered solution was evaporated at a temperature of $225^{\circ} \mathrm{C}$ for 3 hours. [13]

To obtain nanoparticles of silicon dioxide (quartz), a sieving was carried out prior to grinding since the material found mixed with particles of the order of millimeters, obtaining particles smaller than $148 \mu \mathrm{m} .100 \mathrm{~g}$ of silicon dioxide, $\mathrm{g}$ of $1 \mathrm{~mm}$ zirconium balls and $100 \mathrm{~g}$ of $2.5 \mathrm{~mm}$ diameter zirconium balls and $50 \mathrm{~g}$ of industrial alcohol were weighed, attrition wet grinding method was used during 30-minute intervals. The ground material obtained and the balls were using the ASTM E-11 N60, N120, N270 sieves, having a wet mixture, the industrial alcohol was evaporated at a temperature of $225^{\circ} \mathrm{C}$ for 3 hours. [12]

The characterization of the size and agglomeration of the DLS and AFM particles from the soil was carried out, since the nanometric size was not obtained, a 10\% solids solution was prepared which was centrifuged for 10 minutes at a speed of $1840 \mathrm{rpm}$, Then Using a $0.1 \mu \mathrm{m}$ Millex filter, the filtered solution was evaporated at $225^{\circ} \mathrm{C}$ for 3 hours.

For the elaboration of the nanofluids, the phase of the materials, the volumetric concentration of the nanoparticles and the required volume of water (demineralized) were defined, $2 \%$ in concentration volume was used due to an increase in the thermal conductivity of the fluid but not it is necessary to have a greater quantity of material as is the case of $3 \%$. The nanoparticles were suspended in demineralized water, after 6 hours the nanofluids were sonicated to have a better dispersion. [2] [10]

The cooling liquids were prepared under the ASTM D-3306 and ASTM D-4985 standards, where the following were used: $950 \mathrm{ml}$ of ethylene glycol, $44.4 \mathrm{ml}$ of liquid additives, $2.3 \mathrm{ml}$ of sodium nitrite and $3.3 \mathrm{~g}$ of benzoate sodium, and $1000 \mathrm{ml}$ of nanofluids. For the characterization of the cooling liquids, an FTIR analysis was carried out. [5] [8]

The refrigerants were placed in a Chevrolet Aveo 1.6 gasoline and electronic injection vehicle from the Technology Transfer Center for Training and Research in Vehicle Emissions Control (CCICEV), in the city of Quito where torque and power tests, emissions were carried out. ASM dynamics and fuel consumption, which allowed determining the mechanical and thermal behavior of the engine when using nanoparticle-altered coolants. [9]

\section{Results and discussion}

The methods used to obtain the nanoparticles of titanium dioxide and silicon dioxide allowed to obtain a particle size with an effective diameter of $93.9 \mathrm{~nm}$ and $96.3 \mathrm{~nm}$ respectively, the results of the DLS analysis are presented in the Figures 1 and 2. It was not possible to obtain smaller particles since the method used for the production of nanoparticles formed agglomerations which had a size greater than $100 \mathrm{~nm}$, when centrifuging and filtering the particles, those with a size smaller than $100 \mathrm{~nm}$ were separated.

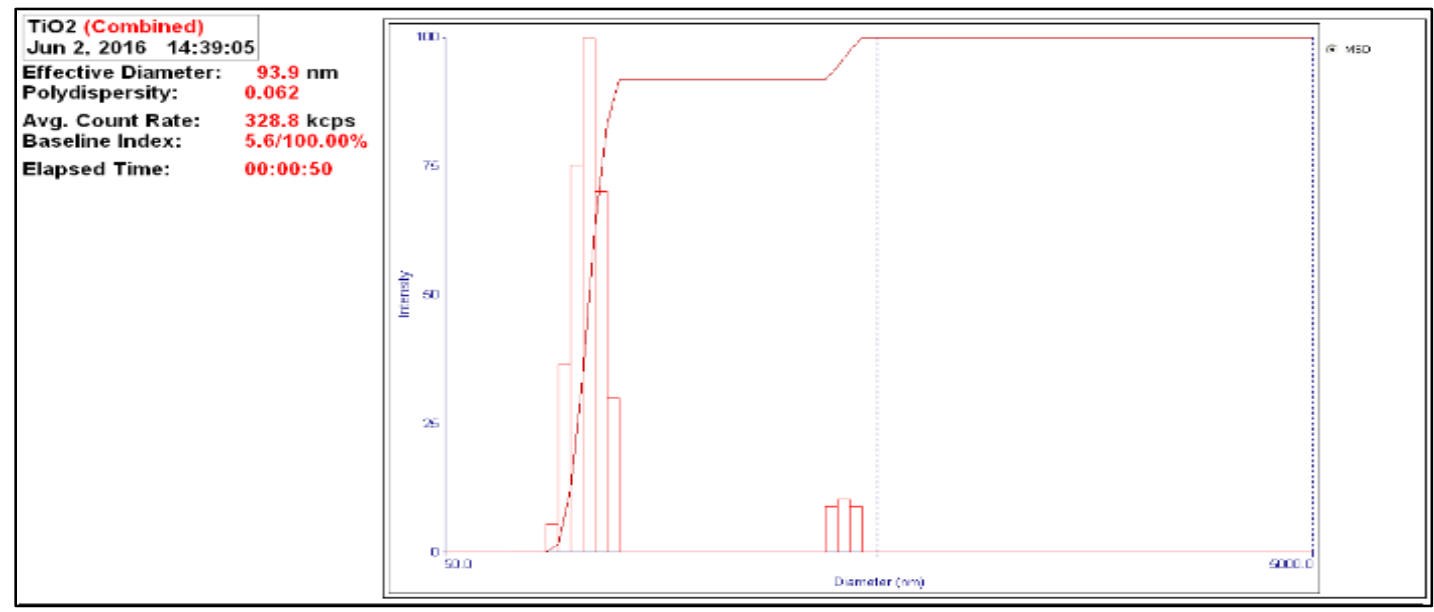

Fig. 1: DLS analysis for a $\mathrm{TiO}_{2}$ sample. 


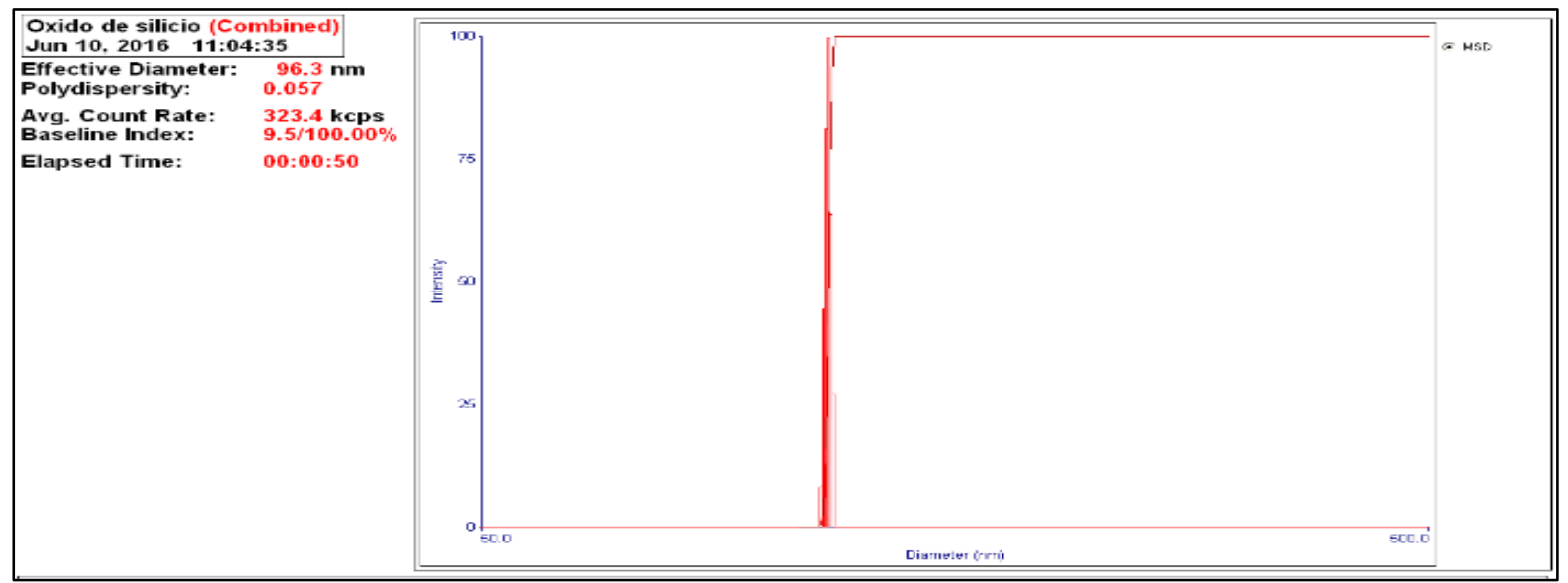

Fig. 2: DLS analysis for a sample of $\mathrm{SiO}_{2}$

It was identified through FT-IR analyzes if there were problems of sulphation, contaminants (water, soot or others), which can alter the performance of the coolants, giving a negative result, which allowed it to be used in the vehicle for the tests. When analyzing the torque and power results, it was determined that the cooling liquid with silicon dioxide nanoparticles had an increase in power of $1 \%$ and $2 \%$ compared to the baseline and titanium, while for the torque or moment values the difference was $3 \%$ between the baseline, titanium and silicon.

This variation can be generated by various factors that are difficult to control, such as: the pressure to which the vehicle is subjected, acceleration speed, power losses generated by the transmission, deformation and pressure of the tires in contact with the rollers, friction of the brakes, temperatures, among others.

All the aforementioned factors are within the percentage of precision of the measuring equipment (3\%), this value when used as a reference for the comparison of the torque and power results allows to say that these are the same, being within the percentage error. The trends of the engine torque and power curves obtained for the three lines of refrigerants are presented in Figures 3 and 4, where it is clear to see that these rise to an almost common maximum point, they present a similar behavior, having a small drop between $2500 \mathrm{rpm}$ and $2700 \mathrm{rpm}$.

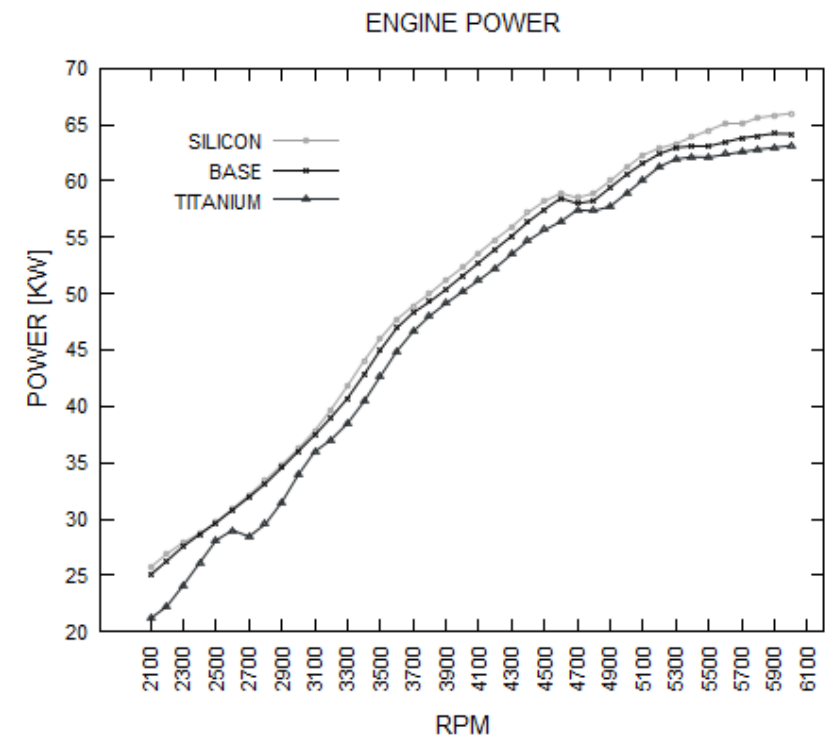

Fig. 3: Characteristic curve of motor power. 


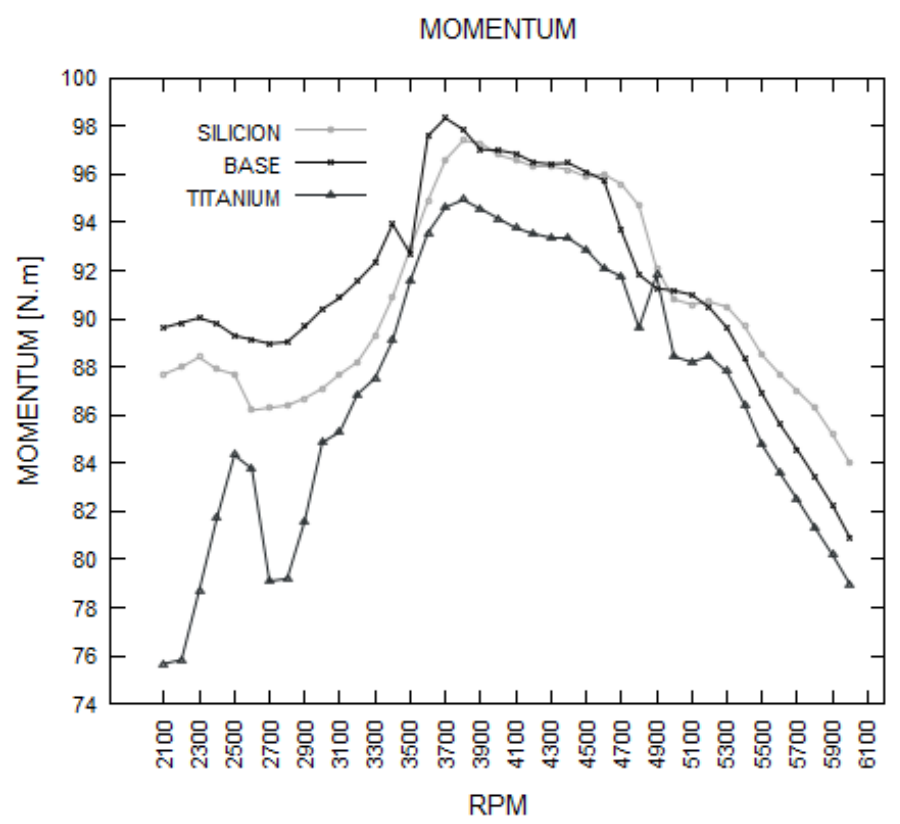

Fig. 4: Characteristic curve of the moment.

The results of the ASM dynamic test are divided into two cycles for this case, since the behavior of the engine was analyzed at different load and speed conditions (ASM 2525 and ASM 5015).For the results of the ASM 2525 cycle, no variation was obtained in the percentage by volume of carbon monoxide, carbon dioxide, and lambda factor for the three refrigerant lines (silicon, titanium and base), since for this analysis it was taken into account the percentage of error of the measuring equipment which is $3 \%$, due to this percentage the values obtained for $\mathrm{CO}, \mathrm{CO} 2$ and $\lambda$ are equal since they are within the appreciation percentage.

The results of the ASM dynamic test are divided into two cycles for this case, since the behavior of the engine was analyzed at different load and speed conditions (ASM 2525 and ASM 5015).For the results of the ASM 2525 cycle, no variation was obtained in the percentage by volume of carbon monoxide, carbon dioxide, and lambda factor for the three refrigerant lines (silicon, titanium and base), since for this analysis it was taken into account the percentage of error of the measuring equipment which is $3 \%$, due to this percentage the values obtained for $\mathrm{CO}, \mathrm{CO} 2$ and $\lambda$ are equal since they are within the appreciation percentage.

For the amounts of ppm of hydrocarbons of the baseline they presented an increase of $50 \%$ and $36 \%$ of ppm compared to the cooling liquids with nanoparticles of titanium and silicon respectively, which means that the combustion for the baseline is more incomplete. A greater increase in hydrocarbons is due to the mixture of combustible air in the combustion chamber for the baseline was a rich mixture greater than that of the titanium and silicon lines.

For the volume of oxygen, the silicon refrigerant liquid presented an increase of $37 \%$ in volume of oxygen compared to the baseline, whereas titanium has the same behavior as the baseline.

Due to the values of the percentage of volume of oxygen of the baseline and titanium are close to 0 , which means the air-fuel mixture is close to the ideal ratio and that the combustion process with the baseline is more efficient since it has the lowest oxygen value. The amounts of ppm of nitrogen oxides, where the cooling liquid with silicon nanoparticles presented an increase of $45 \%$ of ppm of NOx compared to the commercial cooling liquid or baseline.

The titanium coolant had the same behavior as the baseline, which means that the combustion chamber was at a higher temperature when the silicon nanoparticle coolant was used, this effect occurred when the engine was subjected to a load of $25 \%$ since when the load on the motor is increased, the refringent liquids present another behavior.

For the results of the ASM 5015 cycle, as in the ASM 2525 cycle, no variation was obtained in the percentage by volume of carbon monoxide, carbon dioxide, and lambda factor for the three refrigerant lines (silicon, titanium and 
base). For the analysis, the percentage of error of the measuring equipment was taken into account, which is $3 \%$, due to this percentage the values obtained for $\mathrm{CO}, \mathrm{CO} 2$ and $\lambda$ are the same since they are within the percentage of appreciation.

The emission results with a 50\% load on the engine show a behavior similar to the conditions of the ASM 2515 cycle, cycle, with the difference in the production of NOx since a higher load generates a higher temperature in the combustion chamber. With respect to carbon monoxide and carbon dioxide, there is no variation in the volume percentage of carbon monoxide and carbon dioxide for the three lines of the refrigerants, the values obtained are the same.

The amounts of ppm of hydrocarbons in the base cooling liquid showed an increase of $48 \%$ and $59 \%$ of ppm generated by the cooling liquids with silicon and titanium nanoparticles, respectively. The cooling liquid with silicon presented an increase of $64 \%$ in volume of oxygen, whereas titanium had the same behavior as the baseline or commercial line.

The amounts of ppm of nitrogen oxides in the base cooling liquid showed an increase of $12 \%$ and $40 \%$ of ppm of NOx generated by the cooling liquids with silicon and titanium nanoparticles respectively. The NOx percentages are lower when nanoparticulate coolants were used, which means that greater cooling was obtained in the combustion chamber.

For the analysis it was taken into account that the Chevrolet Aveo 1.61 vehicle has a fuel consumption of $8.71 \mathrm{t} / 100 \mathrm{Km}$. The cooling liquid with silicon nanoparticles had 5\% higher performance compared to the baseline or commercial, this is because the line with silicon has a greater cooling of the combustion chamber compared to the baseline.

The cooling liquid with titanium nanoparticles had $16 \%$ higher performance compared to the baseline or commercial, this is because the line with silicon has a greater cooling of the combustion chamber compared to the baseline. The cooling liquid with titanium nanoparticles had $17 \%$ higher performance compared to the silicon line, it could be said that the titanium nanoparticles were dispersed in a better way which caused a better heat transfer, better results should have been obtained with silicon coolant as it has a higher thermal conductivity compared to titanium.

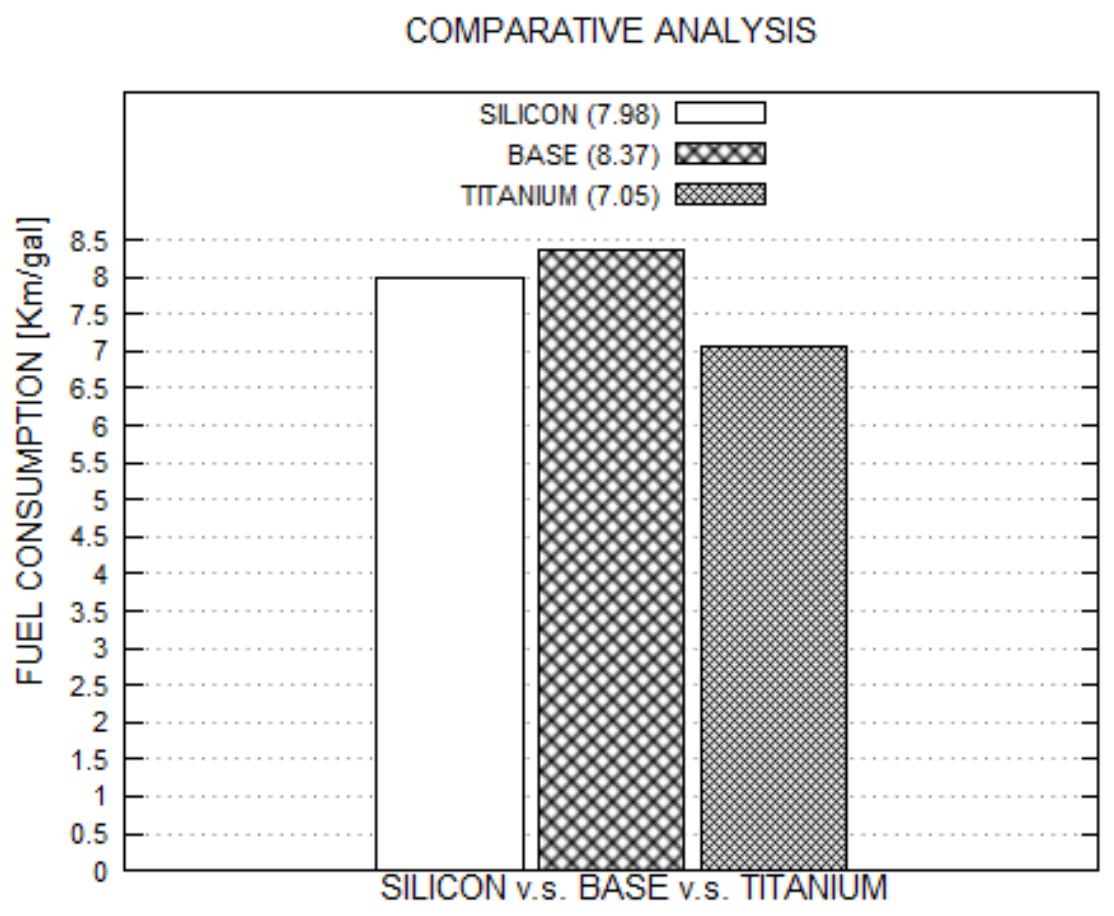

Fig. 5: Comparative performance analysis. 


\section{Conclusions}

The present work presents the results obtained from the evaluation of coolants with silicon and titanium nanoparticles in a Chevrolet Aveo 1.6-liter sedan car. The notable findings are consolidated as follows:

- Two nanofluids were produced based on nanoparticles of titanium dioxide and silicon dioxide with a size of $93.9 \mathrm{~nm}$ and $96.3 \mathrm{~nm}$ respectively, which were taken as base liquids to produce refrigerants.

- No change was obtained in the torque and power values, there was a small variation that is within the precision or error parameters of the measurement equipment, with which it can be said that these values are the same.

- Carbon monoxide (CO) and carbon dioxide (CO2) emissions remained constant for the two loading conditions ASM 2525 and ASM 5015.

- There were no significant lambda variations for the three refrigerant lines under the ASM 2525 and ASM 5015 load conditions.

- When the engine is subjected to greater stress, which means that there is greater combustion in the engine, there is a decrease in NOx emissions (greater cooling) using the refrigerant lines with titanium and silicon nanoparticles.

- An improvement in engine performance was obtained when using coolants with nanoparticles of titanium dioxide and silicon dioxide, the greatest increase was achieved with the titanium line.

\section{References}

[1] S. K. Das, S. U. S. Choi, andW. Yu, Pradeep, Nanofluids Science and Technology, John Wiley \& Sons, 2017.

[2] S. Bobbo, L. Fedele, A. Benetti, , L. Colla, M. Fabrizio, C. Pagura, S. Barison, "Viscosity of water based SWCNH and TiO2 nanofluids," Experimental Thermal and Fluid Science, vol. 36, pp. 65-71, 2015.

[3] A. E. Bergles, "Heat transfer enhancement - the encouragement and accommodation of high heat fluxes," Transaction ASME, Journal Heat Transfer, vol. 119, no. 1, pp. 8-19, 1997.

[4] P. K. Sarma, T. Subramanyam, P. S. Kishore, V. D. Rao, and S. Kakac, "Laminar convective heat transfer with twisted tape inserts in a tube," International Journal of Thermal Sciences, vol. 42, no. 9, pp. 821-828, 2015.

[5] W. Duangthongsuk and S.Wongwises, "An experimental study on the heat transfer performance and pressure drop of TiO2- water nanofluids flowing under a turbulent flow regime," International Journal of Heat and Mass Transfer, vol. 53, no. 1-3, pp. 334-344, 2017.

[6] X. Wang, X. Xu, and S. U. S. Choi, "Thermal conductivity of nanoparticle-fluid mixture," Journal of Thermophysics and Heat Transfer, vol. 13, no. 4, pp. 474-480, 1999.

[7] R. S.Vajjha and D.K .Das, "Experimental determination of thermal conductivity of three nanofluids and development of new correlations," International Journal of Heat and Mass Transfer, vol. 52, no. 21-22, pp. 4675-4682, 2019.

[8] A. Bejan, Convection Heat Transfer, JohnWiley \& Sons, 3rd edition, 2015.

[9] J. A. Eastman, S. U. S. Choi, S. Li, W. Yu, and L. J. Thompson, "Anomalously increased effective thermal conductivities of ethylene glycol-based nanofluids containing copper nanoparticles,” Applied Physics Letters, vol. 78, no. 6, pp. 718$720,2016$.

[10] X. J.Wang and X. F. Li, "Influence of $\mathrm{pH}$ on nanofluids' viscosity and thermal conductivity," Chinese Physics Letters, vol. 26, no. 5, Article ID 056601, 2019.

[11] H.A. Mohammed, H. A. Hasan, and M.A.Wahid, "Heat transfer enhancement of nanofluids in a double pipe heat exchanger with louvered strip inserts," International Communications in Heat and Mass Transfer, vol. 40, pp. 36-46, 2016.

[12] S.Vajjha andD.K.Das, "Experimental determination of thermal conductivity of three nanofluids and development of new correlations," International Journal of Heat and Mass Transfer, vol. 52, no. 21-22, pp. 4675-4682, 2019.

[13] M. N. Pantzali, A. G. Kanaris, K. D. Antoniadis, A. A. Mouza, and S. V. Paras, "Effect of nanofluids on the performance of a miniature plate heat exchanger with modulated surface," International Journal of Heat and Fluid Flow, vol. 30, no. 4, pp. 691-699, 2019.

[14] C. Ho, W. Liu, Y. Chang, C. Lin, 2010. Natural convection heat transfer of alumina water nanofluid in vertical square enclosures: an experimental study. International Journal of Thermal Sciences, 49: 1345-1353. 
[15] P. Namburu, D. Kulkarni, A. Dandekar, D. Das, 2007. Experimental investigation of viscosity and specific heat of silicon dioxide nanofluids. IET Micro and Nano Letters, 2: 67-71

[16] S. Sen Gupta, V. Manoj, S. Krishnan, T. Sreeprasad, P. Singh, T. Pradeep, S. Das, 2011. Thermal conductivity enhancement of nanofluids containing graphene nanosheets. Journal of Applied Physics, 110: 084302-084306. 